\title{
Harald Wolf
}

\section{Gesellschaftskritik und imaginäre Institution Zur Aktualität von Cornelius Castoriadis}

\author{
„Das Motto lautete: 'Anwesend, aber unsichtbar.'“ \\ Thomas Pynchon, Gegen den Tag
}

Gesellschaftskritik ist heute wieder an der Zeit. Vor dem Hintergrund der gesellschaftlichen „Vielfachkrise“ (Demirović et al. 2011) und einer Protestwelle von Kairo bis New York ist der Ruf nach „Wiederbelebung“ und „Rückkehr der Kritik“ (Dörre et al. 2009: 12f.) lauter geworden. Durch Wiederaneignung und Zusammenführung der Traditionslinien kritischer Theorie, so wird gefordert, soll Gesellschaftskritik reanimiert und zu einer Hauptaufgabe der Soziologie gemacht werden (ebd.). Auch Gesellschaftskritik selbst ist zum intensiv bearbeiteten soziologischen und philosophischen Forschungsfeld avanciert, das vermessen, kartiert und evaluiert wird (Jaeggi/Wesche 2009; Iser 2011a). Und die systemstabilisierende Funktion einer vereinnahmten Kritik ist - wie in der Diskussion über den neuen Geist des Kapitalismus (Boltanski/Chiapello 1999) - ebenfalls ein wichtiger Topos.

Der Name Castoriadis taucht bei solchen Wiederbelebungen, Vermessungen und Funktionsbestimmungen von Kritik - verglichen mit sozialtheoretischen Galionsfiguren wie Habermas oder Honneth, Foucault oder Bourdieu - so gut wie nicht auf. Wird er einmal am Rande flüchtig erwähnt, dann meist in wenig erhellender Weise. Der vorliegende Aufsatz versucht hingegen die Aktualität von Castoriadis zu demonstrieren, indem er auf Aspekte seines Werks hinweist, die für die Diskussionen über die Rolle von Kritik „anschlussfähig“ sind und zugleich Schwachstellen dieser Diskussionen kenntlich machen. Die Hinweise sollen zugleich zeigen, dass Castoriadis einen wertvollen Beitrag zur Neuorientierung kritischen Denkens und emanzipatorischen Handelns leistet, der mehr Aufmerksamkeit verdient und dabei helfen könnte, sich im Labyrinth von „Vielfachkrise“ und Kritik nicht wieder in alten Blindgängen linker Theorie und Politik zu verirren.

Einleitend skizziere ich einige Wegmarken der Denkbewegung von Castoriadis und die Konturen von ihm geschaffener Denkfiguren zum Verständnis des 
Gesellschaftlich-Geschichtlichen (1.). Anschlussmöglichkeiten an die Diskussionen über Gesellschaftskritik erläutere ich dann in drei Schritten. Erstens fällt mit jenen Denkfiguren ein anderes Licht auf die Bedingungen der Möglichkeit und die Möglichkeiten der Begründung von Kritik (2.).Zweitens setzt Castoriadis bei der Betrachtung des Kapitalismus andere Akzente als gegenwärtig dominierende Spielarten der Analyse und Kritik. Das betrifft vor allem deren Tendenz, Kapitalismuskritik primär als Marktkritik zu konzipieren, während Castoriadis den Kapitalismus als Organisations-, Rationalisierungs- und Kontrollprojekt begreift und dadurch andere Kritikperspektiven eröffnet (3.). Daraus folgt drittens das ausgeprägte Interesse an Widerstand und praktischer Kritik im Alltag, die als Keime autonomer Vergesellschaftung gedeutet werden (4.). Ein kurzer Ausblick umreißt Fluchtlinien von Gesellschaftskritik heute (5.). ${ }^{1}$

\section{Magma Castoriadis: Vom Marxismus zur imaginären Institution}

Es fiel von Anfang an schwer, Castoriadis in die herrschenden Diskurskonjunkturen einzuordnen. Die theoretischen und politischen Barrieren, die einer angemessenen Rezeption in Frankreich im Weg standen (Breckman 2012), waren bislang auch hierzulande hoch. Vorübergehende Resonanz fanden seine Arbeiten nach Veröffentlichung der deutschen Übersetzung des Hauptwerks Gesellschaft als imaginäre Institution im Jahr 1984 (Habermas 1985: 380-389; Honneth 1985; Brunkhorst 1986; Joas 1989). Aber die Zeit der Diskussion und des Subsumierens - mit von Habermas und Honneth geprägten Spielmarken wie „Praxisphilosophie“, „fundamentalontologisch“, „Rettung der Revolution“, „Kosmologie“, „Welterschließung“, „lebensphilosophisch“ - war nur kurz, Castoriadis rasch ad acta gelegt. ${ }^{2}$ Der politisch-historische Kontext seiner Denkbewegung war kaum wahrgenommen worden, was zu den ungenauen Charakterisierungen beitrug, die gerade das Originelle - im Doppelsinn von Neuanfang und Eigenständigkeit - an ihr verdeckten; ihr un-, ja antiakademischer (und auch noch provokativ antimarxistischer) Charakter blieb ohnehin suspekt (Wolf 1998).

1 Bei Silke van Dyk und Martin Kronauer bedanke ich mich für hilfreiche Kritik und viele Überarbeitungsvorschläge, die ich fast alle einleuchtend fand und versucht habe, aufzugreifen.

2 Eine wichtige Ausnahme bilden die Beiträge von Arnason (1988; 2012), die aber vor allem im Rahmen der breiteren englischsprachigen Rezeption wegweisend waren. In den Kanon zeitgenössischer Sozialtheorie aufgenommen haben ihn hierzulande explizit, so weit ich sehe, nur Joas/Knöbl (2004). Die internationale Castoriadis-Rezeption ist in den Bibliographien der "Cornelius Castoriadis/Agora International Website“ gut dokumentiert (http://www.agorainternational.org/). 
Der revolutionäre Marxismus war Ausgangspunkt und Ferment jener Denkbewegung. Die „Urerfahrung“ (Arnason) von Castoriadis war die Katastrophengeschichte der 1930er und 1940er Jahre, die in Griechenland, wo er aufwuchs und früh in der extremen Linken aktiv war, Ende 1944 in einem stalinistischen Umsturzversuch kulminierte. Das war nicht nur die Geschichte von Faschismus, Weltkrieg und Holocaust, sondern auch des Albtraums der Verwandlung des revolutionären Marxismus in ein neues totalitäres Terror- und Ausbeutungsregime. Dem griechischen Bürgerkrieg glücklich entronnen und seit 1945 in Frankreich lebend, sah Castoriadis nun die Hauptaufgabe darin, das von Auslöschung bedrohte revolutionäre Erbe zu retten und weiterzuentwickeln. Seit 1949 analysierte und kritisierte er mit der heterodoxen Gruppe „Socialisme ou Barbarie“ den östlichen wie westlichen „bürokratischen Kapitalismus“, den traditionellen Marxismus und Marx selber (Castoriadis 1972). Nach dem Ende von „Socialisme ou Barbarie“ Mitte der 1960er Jahre (zur Geschichte der Gruppe: Gabler 2009) war er, mit einer kurzen Ausnahme im Pariser Mai 68 (vgl. Castoriadis 2009), nicht mehr direkt politisch aktiv. Der Bankrott der überlieferten Sozialtheorien machte nach seiner Ansicht „eine Neubetrachtung des gesamten gedanklichen Universums, in dem die politische Emanzipationsbewegung seit Jahrhunderten angesiedelt ist, notwendig", und an dieser ambitionierten Aufgabe arbeitete er den Rest seines Lebens (Castoriadis 1994: 19).

Die Wiederaufnahme des revolutionären Erbes hatte keine Konservierung, sondern eine Neukonzeption zum Ziel, die Castoriadis von Anfang an im Bewusstsein schwerwiegender Ambivalenzen dieses Erbes verfolgt. Er erkennt im Marx'schen Theorieprojekt - ähnlich wie andere Vertreter des „westlichen Marxismus" - die problematische Vermischung zweier konträrer Elemente: einerseits das Streben nach dem allumfassenden und abgeschlossenen System einer objektiven, deterministischen wissenschaftlichen Analyse und Theorie von Gesellschaft und Geschichte; andererseits die Entdeckung des Klassenkampfs als einer „praktisch-kritischen Tätigkeit“ der revolutionären Veränderung oder, in den Worten von Castoriadis: der „schöpferische[n] Aktivität dutzender Millionen von Menschen, die sich während und nach der Revolution entfaltet und deren revolutionärer und kosmogonischer Charakter genau darin besteht, dass ihr Inhalt ursprünglich und unvorhersehbar sein wird" (Castoriadis 1952: 145; Übersetzung durch mich, H.W.).

Castoriadis entwickelt seinen Neuentwurf als Kritik des - dominanten und politisch fatalen - ersten Elements und als gründliche, den ursprünglichen Marx'schen Rahmen schließlich sprengende, Ausarbeitung des zweiten Elementes. Auch wenn er alsbald von einem Zusammenbruch des Marxismus spricht und von der Alternative, Marxist oder Revolutionär zu bleiben (Castoriadis 1975: 28), behalten wichtige Einsichten von Marx für ihn ihre Bedeutung, denen er 
indes eine ganz neuartige Wendung gibt. Den Kern der Intuitionen, die ihn bei der Ausarbeitung des zweiten Elementes leiten, bildet die doppelte Idee vom Sozialismus als „Freisetzung des schöpferischen Handelns der unterdrückten Massen“ (Castoriadis 1955: 75) und als „Autonomie, als freies [...], sich selbst bestimmendes Handeln, als Arbeiterverwaltung in allen Bereichen “ (ebd.: 86). Beharrlich versucht er, „die Konsequenzen dieser Idee in alle Richtungen zu entwickeln“(ebd.: 75). Der Autonomiegedanke wird zum Dreh- und Angelpunkt seines Begriffes von Praxis. Und den Gedanken des kollektiven schöpferischen Handelns überführt er - verallgemeinert und von normativen Konnotationen befreit - in das Konzept des gesellschaftlichen Imaginären.

Seinen Begriff der Praxis reserviert Castoriadis für dasjenige Tun, „worin der oder die anderen als autonome Wesen angesehen und als wesentlicher Faktor bei der Entfaltung ihrer eigenen Autonomie betrachtet werden." (Castoriadis 1975: 128) Individuelle Autonomie und Freiheit werden somit von vornherein als notwendig gesellschaftlich vermittelte Bestimmungen gedacht: „, $[\mathrm{M}]$ eine Freiheit beginnt dort, wo die Freiheit des anderen beginnt.“ (ebd.: 158) Zur Praxis gehören die revolutionäre Politik, ,die sich mit der Organisation und Orientierung der Gesellschaft auf die Autonomie aller hin befaßt“ (ebd.: 132), die wahre Pädagogik und die echte Medizin (einschließlich der Psychoanalyse) (ebd.: 128). Deren „Orientierung auf Autonomie“, der Autonomieentwurf, hat eine wichtige theoretische Implikation: die Absage an die Illusion eines abgeschlossenen Wissens und eine spekulative Theorie, die ihren Gegenstand nur als vollendeten denken kann, während es Autonomie nur gibt, wenn ihr Gegenstand (Ziele, Mittel) „seinem Wesen nach jeden Abschluß überschreitet und wenn sie selbst in einem ständig sich wandelnden Verhältnis zu diesem Objekt steht" (ebd.: 153).

Das Verhältnis von Erkenntnis und Praxis versucht Castoriadis nun, im Unterschied zu ähnlich gelagerten Bemühungen der Frankfurter Schule, durch die Betrachtung der besonderen Seinsweise des Gesellschaftlich-Geschichtlichen (und des Psychischen) weiter aufzuklären. Durch diese ontologische Wendung gelangt er zu neuen Denkfiguren, die der Selbstaufklärung der Praxis - und von Theorie und Kritik als deren Momente - dienen: vor allem den Figuren der Selbstschöpfung des Gesellschaftlich-Geschichtlichen als und durch die imaginäre Institution sowie der Seinsweise des Magmas.

Das Imaginäre - als radikale Einbildungskraft der Psyche wie als radikales Imaginäres einer Gesellschaft und ihrer Institutionen - steht im Mittelpunkt des Neuentwurfs (Arnason 2012). Ebenso wie das Psychische, so erweist sich auch das Gesellschaftlich-Geschichtliche als Seinsweise, deren besondere Aspekte zumeist ausgeblendet bleiben. Sie ist weder als „Subjekt“, noch als „Ding“, noch als „Begriff“ - mit den traditionellen Denkfiguren also - hinreichend zu fassen. Die sich selbst schöpfende (instituierende) und in jeweils spezifischen Institutionen 
verkörperte (instituierte) Gesellschaft ist „radikales Imaginäres“: Grundvermögen der Schöpfung und Setzung gesellschaftlicher imaginärer Bedeutungen durchs anonyme Kollektiv, getragen und am Leben erhalten von Institutionen - damit unsichtbarer, aber überall anwesender Identitätsstifter einer bestimmten Gesellschaft und eines bestimmten Typus von Individuen, die diese Gesellschaft reproduzieren.

Das Imaginäre bedeutet hier also nicht Ideologie, nicht das, was ablenkt und irreführt, das Fiktive oder das Spiegelbild (wie bei Lacan), sondern „wesentlich indeterminierte Schöpfung von Gestalten/Formen/Bildern, die jeder Rede von 'etwas' zugrundeliegen. Was wir 'Realität' oder 'Rationalität' nennen, verdankt sich überhaupt erst ihnen" (Castoriadis 1975: 12). Sie sind das Konstitutive und Anwesend-Abwesende, das auf die Frage 'Was ist diese Gesellschaft, was macht sie, was bildet die Bedingungen für ihr So-Sein?' immer und in allem wie selbstverständlich antwortet. Die imaginären Bedeutungen, die solche Antworten geben - ein religiöser Götter- oder Gotteskomplex, rationale Organisation, Markt, Wahrheits- und Autonomiestreben -, müssen vom anonymen Kollektiv hervorgebracht und instituiert worden sein, damit Individuen entsprechend geformt werden und sich orientieren und handeln können. In den meisten Gesellschaften verselbständigt sich dieses Imaginäre, regiert sie wie von außen, als fraglose, unbeeinflussbare Pseudonatur. Nichts anderes ist für Castoriadis nun der Kern von Entfremdung bzw. Heteronomie: Verselbständigung der instituierten Bedeutungen gegenüber der sie instituierenden Gesellschaft.

Damit modifiziert sich der Sinn von Autonomie zur spezifischen Form der gesellschaftlichen Selbsterzeugung und -verwandlung, zum neuen Modus des Verhältnisses der Gesellschaft zu ihren Institutionen: die Beseitigung der Heteronomie des Imaginären und seine bewusste Aneignung durch die Gesellschaft. Durch den so gefassten Autonomieentwurf einer reflektierten und selbstkritischen Selbst-Institution erweitert sich das frühere, am Leitfaden des Praxisbegriffs entwickelte Verständnis von sozialer Emanzipation. Diesem Autonomieentwurf ordnet Castoriadis in der Epoche der kapitalistischen Moderne bürgerliche Freiheitsbewegungen und die alte revolutionäre Arbeiterbewegung ebenso zu wie die neuen Frauen-, Studenten-, Jugend- und Ökologiebewegungen und die parallelen Neuanfänge in Kunst, Philosophie und Wissenschaft.

Als Schöpferisches konfrontiert uns das Imaginäre mit der indeterminierten und a-rationalen Dimension der Realität und mit der Frage, wie nicht-mengenförmige, nicht-identitätslogische Aspekte des Seins denkbar und verständlich zu machen sind. Die paradoxe Aufgabe, mit den formalen Mitteln der Sprache das Nicht-Formalisierbare zu denken, versucht Castoriadis zu lösen, indem er, in Auseinandersetzung mit der neueren Mathematik, eine Logik des „Magmas“ konzipiert, die doch etwas „Positives“ über die kreativen, eruptiven, „heißen“ 
Potentiale des Seins aussagen soll (Castoriadis 1981). Auch gesellschaftliches Sein ist zwar offensichtlich „Identität“ und „Menge“ („besteht“ aus bestimmten Individuen, Einkommensklassen, Jahreszahlen, Scheidungsraten...), aber eben nur partiell; zugleich ist es unablässige Schöpfung und Zerstörung im Magma nicht-identischer imaginärer Bedeutungen. Abgrenzung und Verschränkung dieser magmatisch-imaginären und der mengen- und identitätslogischen Dimension zugleich zu denken, ist die Aufgabe: Unvermeidlichkeit der formalisierenden Aktivität der klassischen Logik des Identitätsprinzips, des Satzes vom Widerspruch und der Mengentheorie und der Kreativität - was eine Kritik der institutionalisierten Vorherrschaft mengen- und identitätslogischer Rationalität in der kapitalistischen Moderne einschließt.

In diesem Sinne ist auch die Denkbewegung von Castoriadis ein Magma (Curtis 2007). Die ursprüngliche Intuition der Autonomie hält sich durch, aber öffnet sich zugleich für andere, weiterreichende Bedeutungen, die es erlauben, neue Aspekte der Praxis zu erhellen. Ein Beispiel dafür ist die Tätigkeit der Kritik, deren Bedingungen und Merkmale besser zu verstehen sind, wenn sie als Moment des Autonomieentwurfs betrachtet werden.

\section{Autonomieentwurf als Kritik}

In der „Metadiskussion“ über die verschiedenen Formen von Gesellschaftskritik wird Castoriadis nur sehr verkürzt rezipiert. In dieser Diskussion werden diese Formen umfassend erschlossen, vermessen, kartiert, etikettiert und verglichen, wobei diese Ordnungsbemühungen teilweise schon Züge einer Kritikverwaltungswissenschaft (vgl. Sommer 2012: 239ff.) angenommen haben. Geläufig ist die Unterscheidung in ideologiekritische, genealogische, welterschließende, konstruktivistische, interpretative und rekonstruktive Kritikansätze (Iser 2011a). Castoriadis gilt als Beispiel welterschließender Kritik (ebd.: 148; Boltanski/ Honneth 2009: 111f.; Boltanski 2010: 151f.). Diese Kritikform - der auch so unterschiedliche Autoren wie Benjamin, Adorno, Derrida, Rorty oder Latour zugeordnet werden - ziele auf die Erschließung „bislang nicht wahrgenommener Aspekte der Welt oder der Möglichkeit der Existenz" und erfinde mit Hilfe „neuer und ungewohnter Metaphern oder künstlerischer Perspektiven“'Visionen von der guten und gerechten Gesellschaft (Iser 2011a: 148f.). Sie soll „von den sozialen Lebensbedingungen eine so radikale Neubeschreibung [liefern], daß schlagartig alles die neue Bedeutung eines pathologischen Zustandes" annimmt (Honneth 2000: 81). Als „ontologische[.] Kritik“an „Überinstitutionalisierung“ und ,an der Rigidität von Institutionen“, die sich nur noch identisch reproduzieren, „ohne der Welt oder dem Nichtidentischen im Sinne von Adorno einen 
Resonanzboden zu geben“ (Boltanski/Honneth 2009: 111), versuche sie „im Namen der Welt" alternative Möglichkeiten von Gesellschaft zu erschließen (ebd.: 112f.). Der Bezugspunkt einer solchen ontologischen Gesellschaftskritik müsste, so Honneth, „etwas sein [...], was dem 'Magma' bei Castoriadis entspricht: die unbestimmbare Welt da draußen, jenseits der sozialen Konstruktionen, aus denen unsere Gesellschaft besteht" (ebd.: 112).

Mit Recht erwidert aber Boltanski: „Der Bezug auf die Welt muss nicht emanzipatorisch sein." (ebd.: 113) Das Magma an sich ist kein sinnvoller Bezugspunkt von Gesellschaftskritik. Wie skizziert, versucht Castoriadis mit dieser Denkfigur die nicht-determinierte und nicht-formalisierbare Dimension alles potentiell Gegebenen denkbar zu machen. In jeder Institution lauert hinter dem Instituierten das Instituierende, aus dessen Bedeutungsmagma neue Bestimmungen von Gesellschaft hervorgehen und entnommen, aber eben nicht bestimmt und deduziert werden können. Ebendies ist Schöpfung im Sinne von Castoriadis, und in diesem Sinne verdankt sich jegliche gesellschaftliche Form und Neuschöpfung dem magmatischen Charakter des Gesellschaftlich-Geschichtlichen, sei es der Faustkeil, die Demokratie, die Arbeiterbewegung oder das KZ. Wie kann sich daraus eine Referenz für Gesellschaftskritik ergeben? Im Namen des Magmas des Imaginären zu sprechen heißt, im Namen von Allem und damit von Nichts und Niemandem zu sprechen.

Der einzig mögliche Ausgangs- und Bezugspunkt von Kritik ist für Castoriadis hingegen der Autonomieentwurf. Allerdings lässt sich diese „klare normative Position“" (Joas/Knöbl 2004: 574) weder aus der Idee der imaginären Selbstschöpfung von Gesellschaft „ableiten“ (ebd.) noch „begründen“ (s.u.), sondern nur einnehmen, weil (und wenn) sie selbst als imaginäre Institution und Option schon vorhanden ist. Gesellschaftskritik ist keineswegs, wie Walzer (1993: 13) meint, so alt wie die Gesellschaft selbst, und wirkliche Kritik ist deshalb auch keine „'natürliche’ soziale Aktivität“" (Dubet 2008: 11). Klage und Beschwerde über Missstände, Streit über rechtmäßige oder unrechtmäßige Behandlung oder Belohnung kann es immer und überall geben. Doch Gesellschaftskritik wird daraus erst, wenn die Gesellschaft für die Missstände verantwortlich gemacht, als ungerecht be- und verurteilt und wenn eine Änderung ihrer Institutionen zur Beseitigung der Missstände in Erwägung gezogen wird - und werden kann, weil die bewusste Selbstveränderung der Gesellschaft bereits eine effektive und legitime Option darstellt.

Diese Fähigkeit und Möglichkeit zur sozialen Selbstreflexion ist nicht naturgegeben, sondern historisch voraussetzungsvoll. In vom Imaginären beherrschten heteronomen Gesellschaften gibt es Glaube und Gewissheit: Besetzung der durch die geltende, meist religiöse 'Sozialtheorie' festgelegten Denkmöglichkeiten. Erst wenn ein Prozess der Infragestellung der Institution, der Aufklärung ihres 
imaginären Charakters und der Selbstinstitution - wie in einigen Städten des antiken Griechenlands und einigen europäischen Gesellschaften seit dem Ausgang des Mittelalters - in Gang gekommen ist, eröffnet sich die Möglichkeit der Partizipation an diesem Prozess. Wir können eine kritische Einstellung gegenüber etablierten Einstellungen und Praktiken annehmen, dem gegenüber, was wir und unsere Gesellschaft fähig sind, zu denken und zu tun; und das tun wir, indem wir neue „Figuren des Denkbaren“ (Castoriadis 1999) wie des „Tubaren“ entwickeln, erproben und reflektieren.

Die normative Position der Autonomie als Bezugspunkt von Kritik lässt sich nicht im üblichen Sinne begründen:

"Auf die Frage: Warum Autonomie?, Warum Reflexion?, gibt es keine grundlegende, keine 'vorgängige' Antwort. Es gibt eine gesellschaftlich-geschichtliche Bedingung: dass Autonomieentwurf, Reflexion, Überlegung, Vernunft bereits erschaffen, bereits vorhanden sind, zu unserer Tradition gehören. Doch diese Bedingung ist keine Begründung." (Castoriadis 1989: 225)

Dies markiert einen klaren Gegensatz zu Ansätzen einer rekonstruktiven Kritik, wie sie, in Anknüpfung an die „methodische Struktur des linkshegelianischen Kritikmodells" (Honneth 2007: 66), in den Sozialtheorien von Habermas und Honneth verfolgt wird. Die normativen Maßstäbe von Gesellschaftskritik sollen hier durch eine „Verknüpfung von immanenten Verfahren und kontexttranszendierendem Rationalitätsbegriff“ rekonstruiert und rational begründet werden: „Die Kritik der Gesellschaft kann sich auf diejenigen Ideale innerhalb der gegebenen Sozialordnung stützen, von denen sich gleichzeitig begründet zeigen läßt, daß sie Ausdruck eines Fortschritts im Prozeß der gesellschaftlichen Rationalisierung sind.“ (ebd.) Das läuft darauf hinaus, „,normative Erwartungen zu explizieren, die in der menschlichen Lebensform als solcher gründen und somit für alle Gesellschaften gelten“ (Iser 2011b: 9).

Für eine solche rekonstruktive Kritik ist die menschliche Geschichte, ganz wie für Hegel und Marx, der „Prozeß der Verwirklichung von Vernunft“ (Honneth 2007: 65). Die Vernunft verkörpert sich nicht mehr, „subjektphilosophisch“, im Weltgeist oder im Proletariat, sondern in unterschiedlichen Figuren von Intersubjektivität: Sprachliche Verständigung bei Habermas oder Anerkennung bei Honneth bilden das Telos. ${ }^{3}$ Doch geht es nach wie vor um „Gattungskompetenzen“, die „im Lauf der Geschichte stets [...] vorhanden waren“, und um den Nachweis, „dass die Veränderungen dieser Kompetenzen als Lernprozess und somit als Zu-

3 Darüber hinaus müsse kritische Theorie, seit sich nämlich gezeigt habe, dass sich die Sinngehalte solcher Ideale ins Herrschaftsförmige verkehren können, auch Motive genealogischer Vernunftkritik ,als eine Art von metakritischem Gesichtspunkt“ zwingend einbeziehen (Honneth 2007: 68). 
nahme von Vernünftigkeit verstanden werden können“" (Iser 2011b: 15). Eine allen Kulturen und Epochen "gemeinsame moralische Tiefengrammatik" soll rekonstruierbar sein, die die zentralen Mechanismen von Kritik, Protest und Empörung freilegt (ebd.: 14). Der Fortschritt der Geschichte besteht in der „Entfaltung eines normativen Gehalts [...], der von Anfang an“ in jener Tiefengrammatik, sei es von Verständigungs-, sei es von Anerkennungsverhältnissen, „angelegt war“ (ebd.: 15f.). Gesellschaftskritik wird so zur zeitlosen menschlichen Kernkompetenz oder gehört gar - durch die „sprachlichen Reproduktionsmechanismen der Gattung“ (Habermas 1981: 533) - zu seiner biologischen Grundausstattung. Im Anfang der Geschichte lag ihr Ende also bereits wieder einmal beschlossen.

Für Castoriadis gibt es weder eine rationale Grundlegung der Vernunft, noch der Freiheit oder der Kritik (Castoriadis 1988: 217ff.). Gewiss gibt es eine vernünftige Begründung, aber sie erfolgt nachträglich und stützt sich bereits auf das, was die Autonomie erst ermöglicht - und zwar die Autonomie als effektive gesellschaftlich-geschichtliche Gestalt: als Demokratie und Philosophie. Erst diese imaginäre Doppelinstitution ständiger Infragestellung bestehender Institutionen und ständigen offenen Fragens und Forschens schafft die Unterscheidung zwischen Faktum und Recht bzw. Rechtsgültigkeit. In heteronomen Gesellschaften gibt es nur faktischen Sinn und faktische Geltung. Aber es gibt nicht die Möglichkeit einer Kritik der Geltung einer Regel. Diese ist vielmehr durchs heteronome Imaginäre (Gott, Heilige Schrift, instrumentelle Vernunft...) fraglos verbürgt.

„Doch wir hinterfragen diese Geltung. Wir werfen die Frage nach der Rechtsgültigkeit dieser Regel auf. Wir fragen uns: Was sollen wir von dieser Regel halten, was sollen wir mit ihr machen? Wir erkennen die unendliche Vielfalt geschichtlicher nomoi und stellen die Frage: Haben alle diese nomoi den gleichen Wert, und welchen nomos sollen wir für uns selbst wollen? Das bedeutet, dass wir die Metakategorie der Rechtsgültigkeit einführen (akzeptieren). Es ist leicht zu zeigen, dass dies dem Einsetzen von Reflexion und Überlegung gleichkommt, beide in einem radikalen Sinne verstanden (dem nämlich, dass sie zum Beispiel und vor allem nicht vor unserer eigenen Institution Halt machen) oder dem, was der vertretbare Inhalt des Begriffs Vernunft ist." (Castoriadis 1989: 218)

Ansätzen rekonstruktiver Gesellschaftskritik droht nicht bloß das Erstarren in „unablässigen Begründungsdiskursen“(Sonderegger 2009: 57). Sie sind auch der vergebliche Versuch, sich in der „Herberge zur Vernunft, die aber, als Glaube an die ewige Ratio, erst auf „halbem Wege zwischen Glaube und Kritik“ liegt (Pessoa 2003: 182), sicher einzurichten. Recht verstandene Reflexion und Kritik bleiben dagegen der stets „,ungewisse Versuch, das Projekt einer Aufklärung der Welt zu verwirklichen. [...] Es ist der Versuch, die Welt zu denken, ohne vorher oder nachher sicher zu sein, daß die Welt sich tatsächlich denken läßt, ja sogar ohne zu wissen, was Denken eigentlich genau heißt." (Castoriadis 1975: 127) 


\section{Das kapitalistische Imaginäre: „Organisation der Welt"}

Weder Autonomie noch Kritik bilden indes in modernen Gesellschaften die „allgemeine Beleuchtung, worin alle übrigen Farben getaucht sind“ (Marx), sondern der Kapitalismus. Und Castoriadis setzt auch mit Blick auf Kapitalismusanalyse und -kritik eigenständige Akzente. Den Kapitalismus versteht er in erster Linie als Kontrollprojekt. Kapitalistische Heteronomie instituiert und entwickelt sich demnach vorrangig in der imaginären Perspektive einer unbegrenzten Ausdehnung (pseudo-)rationaler (Pseudo-)Kontrolle. Nicht Markt und Tausch, wie bei heute vorherrschenden Varianten der Kapitalismusanalyse, sondern Kontrolle und Organisation rücken ins Zentrum. Das kapitalistische Imaginäre einer „Organisation der Welt“ (Türk 1995) treibt ein widersprüchliches Kontrollprojekt an, das unkontrolliert zwischen Ausschluss und Einschluss der (oder des) Beherrschten hin- und herpendelt (Wolf 1999). Auf diesem schwankenden Grund entwickelt sich eine beispiellose Entfesselung von technischen wie organisatorischen Produktiv- und Destruktivkräften, die mehr und mehr die Form von ökonomischen, politischen und ökologischen Katastrophen annimmt.

Wer vom Kapitalismus spricht, darf also von der Organisation nicht schweigen. Bereits Benjamin hatte „die Organisation [als] das eigentliche Medium“ bezeichnet, „in welchem die Verdinglichung der menschlichen Beziehungen sich abspielt - das einzige übrigens auch in dem sie könnte überwunden werden“" (Benjamin 1930: 423). In diesem Sinne nicht bloß Markt-, sondern vor allem Organisationskritik zu betreiben, fordert Castoriadis spätestens seit Anfang der 1960er Jahre:

„Die zentrale Kategorie, um die kapitalistischen gesellschaftlichen Beziehungen zu verstehen, war für Marx die der Verdinglichung, die sich aus der Umwandlung aller menschlichen Beziehungen in Marktbeziehungen ergab. Für uns ist das zentrale strukturierende Moment der heutigen Gesellschaft nicht der Markt, sondern die bürokratisch-hierarchische Organisation." (Castoriadis 1963: 150).

Im Kapitalismus hat die Institution der „Arbeitsorganisation“ andere, direkt marktvermittelte oder gemeinschaftliche Formen der Leistungserbringung und -koordination erfolgreich verdrängt oder marginalisiert. Angesichts ihrer Allgegenwart müsste jedem außerirdischen Beobachter irdischer Gesellschaftsstrukturen die Rede von einer "Marktwirtschaft“ absurd erscheinen (Simon 1996). Kapitalistische Arbeitsorganisation ist zur dominanten Regulierungs- und Nutzungsform kollektiver Unternehmungen und Tätigkeiten geworden. Sie erfüllt im gesellschaftlichen Zusammenhang eine zentrale intermediäre Funktion: Sie dient als der ,programmatisch-herrschaftliche Filter oder [...] Destillierkolben zwischen lebendiger Ko-Operation und real-abstraktiven Systemen“" (Türk 1995: 201). Indem die Institutionen der Wirtschaft, Politik, Bildung oder Wissenschaft sich „real-abstrahierend“ auf materielle bzw. lebensweltliche Umwelten als Basis 
beziehen, haben die Bezüge einerseits ideologischen Charakter und bedürfen andererseits immer der Spezifikation und Operationalisierung in Form von „Programmen“, die die Kopplung zwischen Institution und (Lebens-)Welt herstellen. Aus solcher aus- und zurichtenden Programmierung besteht der Betriebsmodus formaler Organisationen. Auch die Unterordnung aller gesellschaftlichen Bereiche unter das kapitalistische Leitprogramm eines rationalisierend-kontrollierenden Zugriffs auf Ressourcen von außen vermittelt sich über organisationale Programme. Arbeitsorganisationen haben somit in der funktional differenzierten kapitalistische Gesellschaft eine Schlüsselrolle: Sie transformieren konkrete Leistungen und Interaktionen in abstrakte „Systemfunktionen“ und umgekehrt, sie verkörpern und reproduzieren Herrschaft und Ungleichheit, und sie verbinden die gesellschaftlichen Teilbereiche untereinander (Türk 1995).

Die These einer solchen Schlüsselrolle kapitalistischer Organisation kann hier sicher nicht hinreichend begründet werden. Erinnert sei stattdessen an die lange, teils marxistische, teils nicht-marxistische Forschungstradition der Marx, Sombart, Veblen, Schumpeter, Keynes, Polanyi, Braudel oder Galbraith, in der auf der institutionellen Einbettung, Überformung und Ersetzung von Markt- durch staatliche wie private Organisationsformen insistiert wird (Boyer 2011) und auf die Castoriadis sich berufen konnte (Castoriadis 1997). Wirtschaftsgeschichtlich betrachtet, das hat vor allem Braudel (1979) - und nach ihm Wallerstein (1995: 248ff.) - herausgearbeitet, betritt der moderne Kapitalismus geradezu als GegenMarkt die Bühne: als obskure Welt weitgespannter, langfristiger Kapitaltransaktionen und komplizierter zukunftsgerichteter Kalkulationsverfahren, die die überschaubare Welt des Marktes und der Konkurrenz zunehmend überschattet und beherrscht. Kapitalismus heißt von Anfang an Konzentration der Mittel, Tendenz zur Monopolbildung und erzwungener und ungleicher Tausch. Kapital ist Macht und Machtsteigerung: durch Kontrolle (und ständige Neuschaffung) von Ungewissheitszonen und mittels Herrschaft durch Organisation (und ständige „finanzgetriebene“ Reorganisation) von Arbeit (Nitzan/Bichler 2009).

Genau dies wird heute oft vergessen. Der Markt ist die Leitsemantik der „Diskursformation“, in der sich die Wiederbelebungsversuche der Gesellschaftskritik bewegen und in der die „Befähigung bzw. Erziehung der Menschen zur 'Marktlichkeit'" die Hauptsorge aller Befähiger und Erzieher geworden zu sein scheint (Dörre et al. 2009: 10f.). Auch nach der "großen Rezession“ der vergangenen Jahre beherrschen die „Marktpropagandisten“ (ebd.: 15) das politisch-ideologische Feld (Crouch 2011). Deren penetrante Rede von der „Marktwirtschaft“ scheint zum einen ein Symptom ideologischer Regression, einer Art Selbstverdummung der Herrschenden, zu sein (Castoriadis 1997); sie erfüllt zum anderen die Funktion, die Bedeutung und Macht von Organisationen zu kaschieren und Realitäten des Systems wie die Herrschaft der Unternehmensbürokratien über die Beschäftigten 
oder der Konzerne über die Konsumenten hinterm Marktschein verschwinden zu lassen. Wenn auch Kapitalismuskritik meint, von einem „neuartigen Marktkapitalismus“(Streeck 2009: 15) sprechen und als dessen „dominante[n] Modus der Vergesellschaftung [den] Markt“ ins Visier nehmen zu müssen, der „[w] ie ein schwarzes Loch [...] sich mit jeder Einverleibung neuer Bereiche [vergrößert]“ (Nachtwey 2010: 371), dann läuft sie erkennbar Gefahr, diesem Schein zu erliegen und der Selbstverdummung der Herrschenden durch „Vermarktlichung“ auch noch des kritischen Diskurses zu folgen. Ein Gutteil der globalen Vielfachprobleme, die in stochastischer Folge in ökonomischen und politischen „Unfällen“ und in "Umweltkatastrophen" aufbrechen und sich aus der unverminderten Wirksamkeit kapitalistischer Organisations- und Kontrolllogik ergeben, wie sie etwa an der dominanten Rolle der internationalen Konzernbürokratien abzulesen ist (Crouch 2011: 79ff.), bleibt so unverstanden.

Im Gegenzug nun einen „organisatorischen Imperialismus“ zu propagieren (wie Perrow 1996), wäre ebenfalls verfehlt. Auch eine Kapitalismusanalyse, die von der Organisation als Schlüsselphänomen ausgeht, muss sich von einem mehrdimensionalen Gesellschaftsverständnis leiten lassen. Dies zum einen, um das historischspezifische und historisch veränderliche Zusammenspiel von gesellschaftlichen Teilbereichen, Organisationsformen und Geldwirtschaft in den Blick zu bekommen, denn dieses Zusammenspiel bestimmt Art, Ausrichtung und Intensität des "Ökonomisierungsdrucks“, der in kapitalistischen Gesellschaften auf sämtlichen Sphären lastet (Schimank 2010), wie des für sie ebenso charakteristischen Innovationsdrucks (Deutschmann 1999). Und zum anderen muss, wiederum Castoriadis folgend, Platz bleiben für ein weiteres wesentliches Komplement der Organisations- und Beherrschungslogik: den notwendigen Einschluss und die Spontaneität der Beherrschten (Wolf 2012). Arbeit in kapitalistischen Arbeitsorganisationen ist nie auf restlos angepasstes, fremdbestimmtes und fremdgesteuertes Verhalten reduzierbar und in diesem Sinne „reell“ unters Kapital zu subsumieren. Ganz im Gegenteil: Für ihr Funktionieren wie für ihre Evolution bleibt unerlässlich, was ihren Bestand gefährden könnte: die Selbsttätigkeit der Arbeitenden.

„[D]ie kapitalistische Organisation der Produktion [...] ist ständig bestrebt, möglichst jeden Arbeiter auf ein rein ausführendes Organ zu reduzieren, würde aber sofort zusammenbrechen, wenn diese Reduktion voll und ganz gelänge: Sie ist also gezwungen, die Ausführenden zur Beteiligung am Produktionsprozess zu ermuntern und ihnen gleichzeitig jegliche Initiative zu untersagen." (Castoriadis 1960/61: 94)

Dieser Konflikt bedeutet zwar nicht zwangsläufig, wie Castoriadis zunächst meinte, dass der durch ihn befeuerte „Klassenkampf der Arbeiter zu einer permanenten und unmittelbaren Infragestellung der Systemgrundlagen " führen muss (ebd.). Er setzt dem kapitalistischen Organisations- und Kontrollprojekt aber unüberschreitbare Grenzen und impliziert profunde Abhängigkeiten. Es 
bleibt immer abhängig von lebendiger Arbeit, deren Lebendigkeit es fürchten muss, und „angewiesen auf ideologische, legitimatorische, qualifikatorische, politische und materielle Ressourcen", die es selbst nicht zu produzieren vermag (Türk 1995: 88). Diese Abhängigkeit wiegt besonders schwer, weil nicht nur rationale Kontrolle, sondern deren grenzenlose Ausdehnung das Ziel ist, was die Besetzung einer spezifischen Form sozialen Wandels einschließt: den Wahn eines inhaltsleeren, immer mehr zu beschleunigenden „Fortschritts" und „Wachstums“ und einer endlosen „schöpferischen Zerstörung“ der sozialen wie der natürlichen Welt. Dafür müssen jene „externen“ Ressourcen immer wieder neu mobilisiert und dafür muss vor allem die Kreativität des Sozialen immer wieder gezielt in Anspruch genommen werden - und zugleich nach Maßgabe der rationalen Kontrolllogik eingedämmt (Arnason 1988: 245ff.).

Diese Doppelcodierung des kapitalistischen Imaginären kommt sowohl in dominanten kulturellen Orientierungen als auch den tragenden Institutionen und „Programmen“ zum Ausdruck und bringt eine widersprüchliche historische Dynamik in Gang. So scheint die Geschichte kapitalistischer Arbeitsorganisationen, wie historisch-soziologische Forschungen nahelegen, zwischen entsprechenden kulturellen Antinomien zu oszillieren: dem Pol der „rationalen“ systemischen Steuerung (z.B. durch den Taylorismus), der Ausschluss und Eindämmung betont, und dem Pol der sozialen Integration (z.B. durch Human Relations oder Unternehmenskultur), der Einschluss und Mobilisierung in den Vordergrund rückt (vgl. Wolf2008). Der besondere Akzent aufEinbindung und Einschluss der Arbeitssubjekte - oder die „Subjektivierung“ - ist deshalb weder ein Spezifikum der gegenwärtigen Entwicklungsphase noch etwa ein reines Diskursphänomen. Er ergibt sich als mögliche Ausdeutung des kapitalistischen Geistes und antwortet auf immanente Organisationsprobleme mit organisatorischen Ideologien und Programmen.

In ihrer prominenten Analyse der Entwicklung des „,neuen Geistes des Kapitalismus" ignorieren Boltanski und Chiapello (1999) hingegen solche immanenten Ursachen weitgehend und übertreiben deshalb die Rolle der externen Kritik. Wie erinnerlich, führen sie die Entstehung dieses neuen Geistes, der um die Metapher des Netzwerks kreist und „Projekt“, „Flexibilität“, „Autonomie“, „Teamspirit“ und „Vertrauen“ positiv konnotiert, in hohem Maße auf die Adaption eines bestimmten Typs von Kapitalismuskritik zurück, der „Künstlerkritik“:

„Sie hat sich zunächst in [...] Künstler- und Intellektuellenkreisen entwickelt und [...] kritisiert die Unterdrückung in einer kapitalistischen Welt (die Herrschaft des Marktes, die Disziplin, die Fabrik), die Uniformierung in einer Massengesellschaft und die Transformation aller Gegenstände in Waren. Demgegenüber pflegt sie ein Ideal individueller Autonomie und Freiheit, ihre Wertschätzung gilt der Einzigartigkeit und Authentizität." (Boltanski/Chiapello 2001: 468) 
Obwohl sie sich aufCastoriadis berufen und die Figur des kapitalistischen Geistes sogar der Einschluss-/Ausschluss-Figur direkt nachbilden (Boltanski/Chiapello 1999: 614, Anm. 54), verfehlen Boltanski und Chiapello deren ganzen Sinn. Sie reduzieren sie nämlich auf die Teilaspekte der Normativität und Legitimität und die Ideale, die - wie jene der von den 1968er-Bewegungen revitalisierten „Künstlerkritik“ - an das als „amoralisch“ und „absurd“ konzipierte kapitalistische Projekt von außen herangetragen und von diesem nur aufgenommen werden (müssen), um Folgebereitschaft und Motivation zu sichern (Boltanski/ Chiapello 2001: 462f.).

Mit Castoriadis ist dagegen auf die enge Verschränkung von Legitimationsaspekten und Kritik mit den Widersprüchen und Funktionsproblemen von Arbeitsorganisationen zu verweisen, die aus der skizzierten Doppelcodierung resultieren. Ohne Einschluss von Subjektivität, Initiative und Kreativität der ihr Unterworfenen war und ist kapitalistische „Organisation der Welt" nie und nirgends realisierbar - er ist vielmehr ihre zentrale innere Funktionsbedingung. Zugleich unterminiert das kapitalistische Projekt mit seinen Ausschlussmechanismen und seinem Herrschaftscharakter diese Funktionsbedingung beständig selbst. Dies im Blick lässt sich auch zeigen, wie sich in der alltäglichen Auseinandersetzung mit diesen Widersprüchen und Problemen bereits praktische Kritik entwickelt, die zum Keim von Autonomieforderungen werden kann.

\section{Keime von Kritik und Autonomie: das Beispiel der Selbsttätigkeit in der Arbeit}

Die kapitalistische Arbeitsorganisation provoziert durch ihre eine, dominante Tendenz, den Ausschluss der Arbeitenden aus der Definition von Zielen und Mitteln des Arbeitens, notwendig Selbsttätigkeit. Ausschließende Herrschaftsförmigkeit und asymmetrische Arbeitsteilung zwischen leitenden und ausführenden Funktionen der Arbeitsprozesse haben Fremdbestimmung - und zugleich die Untauglichkeit vorgegebener Regeln, Lücken, Störungen und Dysfunktionen als Alltags- und Dauerphänomen zur Folge. Durch selbsttätiges, von der offiziellen Definition nicht abgedecktes bzw. vorgesehenes Arbeitshandeln müssen die Lücken geschlossen und die Regeln umgangen werden, um die Arbeitsprozesse am Laufen zu halten. Vom aktiven Mitmachen auch gegen die Regeln (oder auch gegen Mitarbeitende) über individuelle kleine Listen bis hin zur geheimen Gegen- und Selbstorganisation sind vielfältige Arten und Muster solch tätigen Eigen-Sinns in der Arbeit möglich und oft beschrieben worden (Wolf 1999: 77ff.; Ackroyd/Thompson 1999). Sie bilden die unkontrollierbaren, oft unsichtbaren Erfahrungsräume einer individuellen und kollektiven Selbsttätigkeit (Wolf2012). 
Notwendige Selbsttätigkeit ist in Arbeitsorganisationen endemisch. Sie ist eingelassen in die Routinen und Rituale des Alltags und wird nicht erst in Ausnahmesituationen wirksam. In der Organisationsforschung werden diese Formen durchaus thematisiert, jedoch nicht als Form von „Kritik“, sondern unter abweichendes Verhalten, implizites Wissen oder Informalität rubriziert. Sie stellen aber die mehr oder - oft - weniger bewusste Auseinandersetzung mit fremdbestimmten Handlungszwängen dar und implizieren die Entscheidung, etwas eigenes und anderes zu tun, als von anderen vorbestimmt. Dabei sind diese Praktiken ambivalent: einerseits weisen sie in Richtung einer Überwindung von Herrschaft und in Richtung von autonomer kollektiver Tätigkeit, andererseits tragen sie zum Funktionieren von Herrschaft bei.

Nur kurz kann mit dem Hinweis auf diese Erfahrungsräume noch der Bogen zu der Diskussion über die Möglichkeiten einer „Soziologie der Kritik“ (Boltanski/Honneth 2009; Boltanski 2010) geschlagen werden, die in eine ähnliche Richtung weist, dabei aber vielleicht noch nicht weit genug geht. Während die meisten bereits erwähnten Kritikformen das Geschäft der Kritik, getreu den Mottos „Ich sehe was, was du nicht siehst“ oder „Denn sie wissen nicht, was sie tun" (Celikates 2009: 39; 60), einem externen Beobachter überlassen, der Gesellschaftsstrukturen als entfremdete, unterdrückende usw. entlarvt, versucht eine "Soziologie der Kritik“ herauszustellen, dass zum Beispiel die Kritik an Organisationen immer auch schon in Organisationen stattfindet und stattfinden muss: als soziale Tätigkeit der Organisationsmitglieder (Celikates 2009: 136ff.; ähnlich: Dubet 2008).

Fragwürdig scheint allerdings die Annahme, dass in den Handlungsmodus der Rechtfertigung und Kritik immer (erst) dann umgeschaltet wird, wenn die Routinen des Alltags aufbrechen und durch Krisen und Konfliktfälle gleichsam ein Rechtfertigungsnotstand entsteht. Nur im Streitfall würden unter Rückgriff auf vorhandene Rechtfertigungsfiguren kritische Argumente mobilisiert (Celikates 2009: 141; inzwischen relativierend: Boltanski 2010: 135ff.). Mit Blick auf eine Kritik im Organisationskontext ist darauf zu verweisen, dass bereits in organisatorische Routinen mehr oder weniger unsichtbare Formen praktischer Organisationskritik einfließen, die - als Schmiermittel organisatorischen Funktionierens und als Interessendurchsetzung der Arbeitenden - bereits diesseits expliziter Konfliktfälle wirksam sind. In dieser Hinsicht wäre eine „Soziologie der Kritik" noch zu radikalisieren.

Kritische Organisations- und Arbeitsanalysen, mit einem Sensorium für die implizite und explizite Selbstorganisation im Rahmen fremdorganisierter Arbeit, erhalten in der von Castoriadis eröffneten Perspektive der Kapitalismusanalyse jedenfalls einen besonderen Stellenwert. Bei „Socialisme ou Barbarie“ wirkte Castoriadis an dem Versuch, Formen und Erfahrungen der Selbsttätigkeit in der 
Arbeit selbsttätig aufzudecken und zum Sprechen zu bringen, maßgeblich mit. Er deutet diese Formen, bei denen die Arbeitenden selbst Entscheidungen gegen offizielle Vorgaben und herrschende Normen treffen, ausführen und verantworten, als praktische Kritik an kapitalistischer Organisation und als Antizipation von Autonomie (Castoriadis 1958; Gabler 2009).

\section{Autonomie und Kritik heute: Endspiele?}

Gesellschaftskritik mit Castoriadis ist unzeitgemäß, und zwar im besten Sinn: als der energische und beharrliche Versuch, gegen die Zeit auf die Zeit für eine kommende Zeit zu wirken (Nietzsche). Die Denkfiguren seines sozialtheoretischen Entwurfs tragen - wie am Beispiel der Kerninstitution kapitalistischer Arbeitsorganisation illustriert - zum besseren Verständnis von Strukturproblemen und Krisenphänomenen der Gegenwartsgesellschaft bei. Sie helfen auch bei der Selbstaufklärung einer sich neu formierenden Kritik. Und sie weisen auf Spuren und Ansätze alternativer, selbstbestimmter Vergesellschaftung im Alltag hin.

Bei Castoriadis selbst wuchs allerdings immer mehr die Skepsis über die Aussichten einer wirklich breiten und durchgreifenden Renaissance des Autonomieentwurfs. Seit dem Abflauen und der Vereinnahmung der neuen sozialen Bewegungen nach 1968 befindet sich dieser Entwurf für ihn im beschleunigten Niedergang. Vorherrschende gesellschaftliche Tendenzen sind der Rückzug ins Private und politische Apathie. Mit der Diagnose vom „Anstieg der Bedeutungslosigkeit“ im neuesten Kapitalismus (Castoriadis 1994) malt er das Bild einer zunehmenden Schwächung elementarer Kapazitäten der gesellschaftlichen Institution und Sinngebung an die Wand, eines Endspiels, das die Bedeutungs- und Machtlosigkeit kritischer Reflexion und der praktisch-kritischen Tätigkeit einschließt. Solche gezielten Übertreibungen sollen wohl auch den Widerstandsgeist wecken, der nötig wäre, die Diagnose zu widerlegen.

Verwirklichte gesellschaftliche Autonomie besteht in der Institutionalisierung bewusster, reflektierter imaginärer Institution. Das Hauptproblem des Autonomieentwurfs bleibt bis heute seine mangelnde Kraft zu solcher Institutionalisierung. Historisch ist er damit immer wieder gescheitert, vereinnahmt oder ins Gegenteil verkehrt worden. Die neuen sozialen Bewegungen nach 1968, als letzte lange historische Welle eines Institutionalisierungsversuchs, bezeugten zwar erneut die nach wie vor vorhandene Macht und Hartnäckigkeit des Strebens nach Autonomie.

„Sie sind aber auch ein Beleg für die Dimension des Scheiterns, die bis heute von den modernen politischen Bewegungen anscheinend nicht zu trennen ist: für die immense Schwierigkeit, der Kritik des Bestehenden eine positive Richtung zu geben, die Unmög- 
lichkeit, das Streben nach Autonomie als zugleich individueller und gesellschaftlicher Autonomie durch die Errichtung einer kollektiven Selbstregierung zu verwirklichen." (Castoriadis 1986: 177)

Die kapitalistische Weltwirtschaft ist heute in großer Unordnung. Nur ein permanentes staatliches Interventions-, Überwachungs- und Sparregime, das selbst zur Unordnung beiträgt, verhindert den Zusammenbruch. Die ökologischen, ökonomischen, politischen und ideologischen Symptome einer „Vielfachkrise“ bleiben aufDauer virulent. Gleichzeitig artikuliert sich aber auch weltweit wachsendes Unbehagen und offene Kritik am Bestehenden. Als Kritik am Markt greift diese Kritik gewiss noch viel zu kurz. Die Widerstände und Proteste gegen die Krisenfolgen haben aber auch Suchbewegungen ausgelöst, die darüber hinausweisen. Die internationale „Occupy“-Bewegung stellt wieder die Forderung - darin an die antiautoritären und antihierarchischen 1968er-Bewegungen anknüpfend (Wallerstein 2011) - nach Formen direkter Demokratie und kollektiver Selbstbestimmung und erprobt sie. Bei libertären Stichwortgebern und Mitinitiatoren der Bewegung sind unschwer Bezüge zu Denkfiguren von Castoriadis auszumachen: Imagination, Schöpfung, Autonomie (Graeber 2012). Vielleicht ist mit dieser erneuten Suche nach Alternativen, die das Bestehende radikal in Frage stellen, endlich doch noch der Moment für das Magma Castoriadis gekommen (Breckman 2012). „Nicht das Bestehende, sondern das, was sein könnte und sein sollte, braucht uns." (Castoriadis 1989: 256)

\section{Literatur}

Ackroyd, Stephen; Thompson, Paul (1999): Organizational Misbehaviour, London.

Arnason, Johann P. (1988): Verständigungsformen und imaginäre Bedeutungen, in: ders., Praxis und Interpretation. Sozialphilosophische Studien, Frankfurt/M., 187-309.

- (2012): Über Marx hinaus - Castoriadis' Ort in der Sozialtheorie, in: Wolf, Harald (Hrsg.): Das Imaginäre im Sozialen. Zur Sozialtheorie von Cornelius Castoriadis. Göttingen (in Vorbereitung).

Benjamin, Walter (1930): Politisierung der Intelligenz.Zu S. Kracauer 'Die Angestellten', in: ders., Angelus Novus. Ausgewählte Schriften 2, Frankfurt/M. 1988, 422-428.

Boltanski, Luc (2010): Soziologie und Sozialkritik. Frankfurter Adorno-Vorlesungen 2008, Berlin.

Boltanski, Luc; Chiapello, Ève (1999): Der neue Geist des Kapitalismus, Konstanz 2003.

- (2001): Die Rolle der Kritik in der Dynamik des Kapitalismus und der normative Wandel, in: Berliner Journal für Soziologie 11 (4), 459-477.

Boltanski, Luc; Honneth, Axel (2009): Soziologie der Kritik oder Kritische Theorie? Ein Gespräch mit Robin Celikates, in: Jaeggi/Wesche 2009, 81-114.

Boyer, Robert (2011): Are there laws of motion of capitalism?, in: Socio-Economic Review 9 (1), 59-81.

Braudel, Fernand (1979): Civilisation matérielle, économie et capitalisme. Tome 2: Les jeux de l'échange, Paris.

Breckman, Warren (2012): Sammelbesprechung der Ausgewählten Schriften von Cornelius Castoriadis, in: Zeitschrift für Ideengeschichte VI (2) (im Erscheinen). 
Brunkhorst, Hauke (1986): Die Zukunft des revolutionären Projekts - Cornelius Castoriadis' Entwurf einer politischen Philosophie, in: Sozialwissenschaftliche Literatur Rundschau 9 (12), 40-45.

Castoriadis, Cornelius (1952): La direction prolétarienne, in: ders., L'expérience du mouvement ouvrier 1. Comment lutter, Paris 1974.

- (1955): Über den Inhalt des Sozialismus I, in: ders., Vom Sozialismus zur autonomen Gesellschaft. Über den Inhalt des Sozialismus. Ausgewählte Schriften 2.1, Lich/Hessen 2007, 65-93.

- (1958): Über den Inhalt des Sozialismus III: Der Kampf der Arbeiter gegen die Organisation des kapitalistischen Unternehmens, in: ders., Vom Sozialismus zur autonomen Gesellschaft. Über den Inhalt des Sozialismus. Ausgewählte Schriften 2.1, Lich/Hessen 2007, 187-243.

- (1960/61): Die revolutionäre Bewegung im modernen Kapitalismus, in: ders., Vom Sozialismus zur autonomen Gesellschaft. Gesellschaftskritik und Politik nach Marx. Ausgewählte Schriften 2.2, Lich/Hessen 2008, 17-144.

- (1963): Die Revolution neu beginnen, in: ders., Sozialismus oder Barbarei. Analysen und Aufrufe zurkulturrevolutionären Veränderung, aus dem Französischen von Jürgen Hoch, Berlin 1980, 145-180.

- (1972), Einleitung, in: ders., Sozialismus oder Barbarei. Analysen und Aufrufe zur kulturrevolutionären Veränderung, Berlin 1980, 9-52.

- (1975): Gesellschaft als imaginäre Institution. Entwurfeinerpolitischen Philosophie, Frankfurt/M. 1984.

- (1981): Die Logik der Magmen und die Frage der Autonomie, in: ders., Das imaginäre Element und die menschliche Schöpfung. Ausgewählte Schriften 3, Lich/Hessen 2010, 109-146.

- (1986): Die Bewegungen der sechziger Jahre, in: ders., Autonomie oder Barbarei. Ausgewählte Schriften 1, Lich/Hessen 2006, 169-181.

- (1988): Individuum, Gesellschaft, Rationalität, Geschichte, in: ders., Das imaginäre Element und die menschliche Schöpfung. Ausgewählte Schriften 3, Lich/Hessen 2010, $189-224$.

- (1989): Getan und zu tun, in: ders., Philosophie, Demokratie, Poiesis. Ausgewählte Schriften 4, Lich/Hessen 2011, 183-269.

- (1994): Der Anstieg der Bedeutungslosigkeit, in: ders., Autonomie oder Barbarei. Ausgewählte Schriften 1, Lich/Hessen 2006, 17-41.

- (1997), Die 'Rationalität' des Kapitalismus, in: Archiv für die Geschichte des Widerstandes und der Arbeit, Nr. 16 (2001), 425-446.

- (1999): Figures du pensable. Les carrefours du labyrinthe VI, Paris.

- (2009): Mai 68: Die vorweggenommene Revolution, Moers.

Celikates, Robin (2009): Kritik als soziale Praxis. Gesellschaftliche Selbstverständigung und kritische Theorie, Frankfurt/New York.

Crouch, Colin (2011): Das befremdliche Überleben des Neoliberalismus. Postdemokratie II, Berlin.

Curtis, David Ames (2007): „Unities and Tensions in the Work of Cornelius Castoriadis, With Some Considerations on the Question of Organization“, http://issuu.com/magmareview/docs/ athens-nostrikeoutword_1_, zuletzt abgerufen: 19.3.2012.

Demirović, Alex; Dück, Julia; Becker, Florian; Bader, Pauline (Hrsg.) (2011): VielfachKrise. Im finanzmarktdominierten Kapitalismus, Hamburg.

Deutschmann, Christoph (1999): Die Verheißung des absoluten Reichtums. Zur religiösen Natur des Kapitalismus, Frankfurt/New York.

Dörre, Klaus; Lessenich, Stephan; Rosa, Hartmut (2009): Soziologie - Kapitalismus - Kritik: Zur Wiederbelebung einer Wahlverwandtschaft, in: dies. (Hrsg.), Soziologie - Kapitalismus - Kritik. Eine Debatte, Frankfurt/M., 9-18.

Dubet, Francois (2008): Ungerechtigkeiten. Zum subjektiven Ungerechtigkeitsempfinden am Arbeitsplatz, Hamburg. 
Gabler, Andrea (2009): Antizipierte Autonomie. Zur Theorie und Praxis der Gruppe „Socialisme ou Barbarie“ (1949 - 1967), Hannover.

Graeber, David (2012): Revolution rückwärts, in: ders., Kampf dem Kamikaze-Kapitalismus. Es gibt Alternativen zum herrschenden System, München, 72-113.

Habermas, Jürgen (1981): Theorie des kommunikativen Handelns I, Frankfurt/M.

- (1985): Der philosophische Diskurs der Moderne. ZwölfVorlesungen, Frankfurt/M.

Honneth, Axel (1985): Eine ontologische Rettung der Revolution. Zur Gesellschaftstheorie von Cornelius Castoriadis, in: ders., Die zerrissene Welt des Sozialen, Frankfurt/M. 1990, 123-143.

- (2000): Über die Möglichkeit einer erschließenden Kritik. Die 'Dialektik der Aufklärung' im Horizont gegenwärtiger Debatten über Sozialkritik, in: ders., Das Andere der Gerechtigkeit. Aufsätze zur praktischen Philosophie, Frankfurt/M., 70-87.

- (2007): Rekonstruktive Gesellschaftskritik unter genealogischem Vorbehalt. Zur Idee der 'Kritik' in der Frankfurter Schule, in: ders., Pathologien der Vernunft. Geschichte und Gegenwart der Kritischen Theorie, Frankfurt/M., 57-69.

Iser, Mattias (2011a): Gesellschaftskritik, in: Göhler, Gerhard et al. (Hrsg.), Politische Theorie. 25 umkämpfte Begriffe zur Einfübrung, 2., aktualisierte und erweiterte Auflage, Wiesbaden, 142-157.

- (2011b): Empörung und Fortschritt. Grundlagen einer kritischen Theorie der Gesellschaft, 2., durchgesehene Auflage, Frankfurt/New York.

Jaeggi, Rahel; Wesche, Tilo (Hrsg.) (2009): Was ist Kritik? Philosophische Positionen, Frankfurt/M.. Joas, Hans (1989): Institutionalisierung als kreativer Prozeß. Zur politischen Philosophie von Cornelius Castoriadis, in: Politische Vierteljahresschrift 30, 585-602.

Joas, Hans; Knöbl, Wolfgang (2004): Sozialtheorie. ZwanzigeinführendeVorlesungen, Frankfurt/M. Nachtwey, Oliver (2010): Legitimationsprobleme des Spätkapitalismus revisited, in: Becker, Karina et al. (Hrsg.), Grenzverschiebungen des Kapitalismus. Umkämpfte Räume und Orte des Widerstands, Frankfurt/New York, 359-379.

Nitzan, Jonathan; Bichler, Shimshon (2009): Capital as Power. A Study of Order and Creorder, New York.

Perrow, Charles (1996): Eine Gesellschaft von Organisationen, in: Kenis, Patrick; Schneider, Volker (Hrsg.), Organisation und Netzwerk. Institutionelle Steuerung in Wirtschaft und Politik, Frankfurt/New York, 75-121.

Pessoa, Fernando (2003): Das Buch der Unruhe des Hilfsbuchhalters Bernardo Soares, Zürich.

Schimank, Uwe (2010): Die funktional differenzierte kapitalistische Gesellschaft als Organisationsgesellschaft - eine theoretische Skizze, in: Endreß, Martin; Matys, Thomas (Hrsg.), Die Ökonomie der Organisation - die Organisation der Ökonomie, Wiesbaden, 33-61.

Simon, Herbert A. (1996), Organisationen und Märkte, in: Kenis, Patrick; Schneider, Volker (Hrsg.), Organisation und Netzwerk. Institutionelle Steuerung in Wirtschaft und Politik, Frankfurt/New York, 47-74.

Sommer, Andreas Urs (2012): Lexikon der imaginären philosophischen Werke, Berlin.

Sonderegger, Ruth (2009): Wie diszipliniert ist (Ideologie-)Kritik? Zwischen Philosophie, Soziologie und Kunst, in: Jaeggi/Wesche 2009, 55-80.

Streeck, Wolfgang (2009): Von der gesteuerten Demokratie zum selbststeuernden Kapitalismus. Die Sozialwissenschaften in der Liberalisierung, in: WestEnd 6 (1), 13-33.

Türk, Klaus (1995), Die Organisation der Welt. Herrschaft durch Organisation in der modernen Gesellschaft, Opladen.

Wallerstein, Immanuel (1995): Die Sozialwissenschaft „kaputtdenken“. Die Grenzen der Paradigmen des 19. Jabrhunderts, Weinheim.

- (2011): The Fantastic Success of Occupy Wall Street, http://www.iwallerstein.com/fantasticsuccess-occupy-wall-street/, zuletzt abgerufen: 19.1.2012. 
Walzer, Michael (1993): Kritik und Gemeinsinn. Drei Wege der Gesellschaftskritik, Frankfurt/M.. Wolf, Harald (1998): 'Die Revolution neu beginnen'. Über Cornelius Castoriadis und Socialisme ou Barbarie, in: Archiv für die Geschichte des Widerstandes und der Arbeit, Nr. 15, 69-112.

- (1999): Arbeit und Autonomie. Ein Versuch über Widersprüche und Metamorphosen kapitalistischer Produktion, Münster.

- (2008): Die duale Institution der Arbeit und der neue(ste) Geist des Kapitalismus. Einige Anmerkungen zu einer Anmerkung, in: Hessinger, Philipp; Wagner, Gabriele (Hrsg.), Ein neuer Geist des Kapitalismus?, Wiesbaden, S. 219-231.

- (2012); Kapitalistische Arbeitsorganisation und Selbsttätigkeit, in: Dörre, Klaus et al. (Hrsg.), Arbeit und Kapitalismustheorie, Frankfurt/New York (in Vorbereitung).

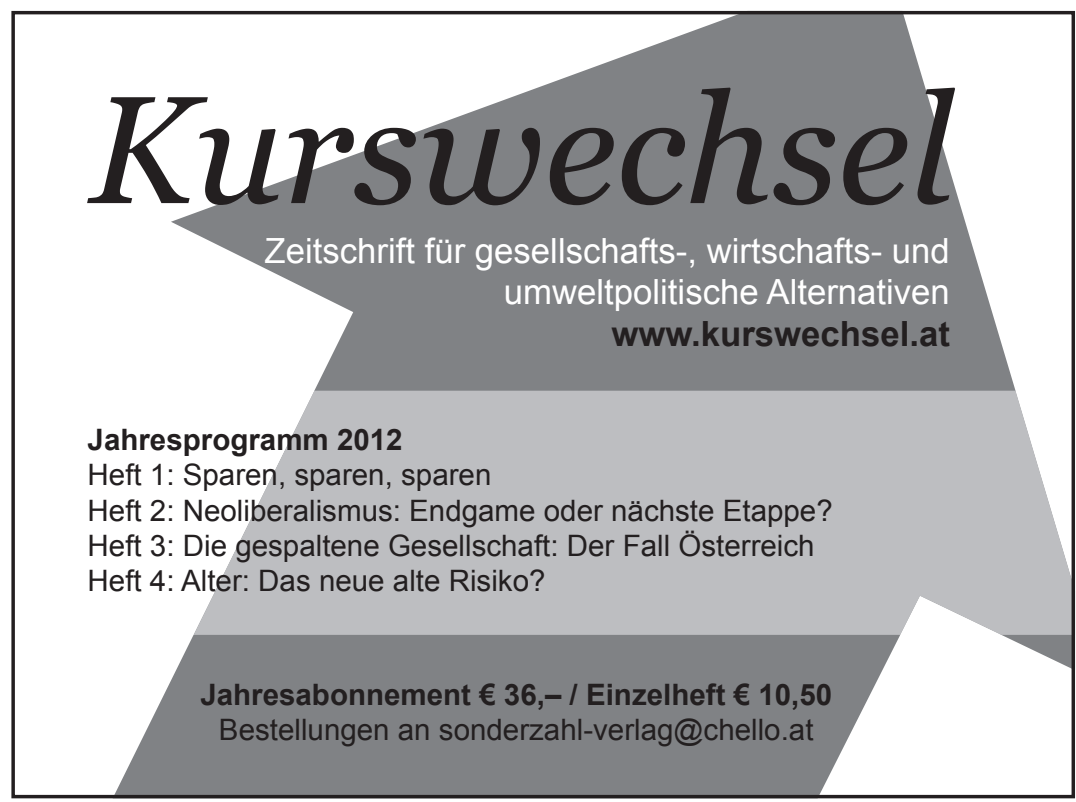

